Check for updates

Cite this: Phys. Chem. Chem. Phys., 2020, 22, 24764

Received 24th May 2020

Accepted 16th October 2020

DOI: $10.1039 / \mathrm{d} 0 \mathrm{cp} 02805 \mathrm{c}$

rsc.li/pccp

\title{
Masking specific effects of ionic liquid constituents at the solid-liquid interface by surface functionalization $\dagger$
}

\author{
Bojana Katana, $\ddagger^{a}$ Dóra Takács $\ddagger^{\mathrm{a}}$ Felix D. Bobbink, (D) ${ }^{\mathrm{b}}$ Paul J. Dyson, ${ }^{\mathrm{b}}$ \\ Nizar B. Alsharif, ${ }^{a}$ Matija Tomšič (D) ${ }^{c}$ and Istvan Szilagyi (D) *a
}

\begin{abstract}
Ion specific effects of ionic liquid (IL) constituents on the surface charge and aggregation properties of two types of particles (positively charged amidine (AL) and polyimidazolium-functionalized sulfate (SL-IP-2) latexes) were investigated in IL solutions containing different anions and the 1-butyl-3-methylimidazolium cation. For the AL systems, the affinity of IL anions to the particle surface followed the sequence chloride $<$ bromide $<$ nitrate $<$ acetate. The critical coagulation concentration values decreased in the same order indicating that ion specific adsorption determines the surface charge density and the extent of the repulsive interparticle forces. In contrast, no tendencies were observed for the SL-IP-2 particles, i.e., both charge and aggregation features were insensitive to the type of anions. This surprising behavior sheds light on that surface functionalization with the polyimidazolium compound effectively masks interfacial ion specific effects. These results indicate new possible routes to the design of processable particle dispersions in ILS irrespective of their composition.
\end{abstract}

\section{Introduction}

Ionic liquids (ILs) continue to attract widespread interest due to their advantageous properties such as low vapor pressure, wide electrochemical window and their capacity to be structurally tuned for specific tasks such as for the functionalization of surfaces. ${ }^{1-3}$ The assembly of the IL cations and anions at solid-liquid interfaces has been extensively studied, since ion specific effects play a major role in the physico-chemical properties of surfaces immersed in ILs or in their solutions. ${ }^{4-6}$ The interfacial structure is responsible for reduced friction between surfaces, ${ }^{7}$ exfoliation of layered materials ${ }^{8}$ and stabilization of various particles in ILs. ${ }^{9-13}$ In addition, specific interactions of IL ions with synthetic and natural macromolecules influence the stability of enyzmes, ${ }^{14}$ the rate of amyloid fibrillization ${ }^{15}$ and phase transition behavior ${ }^{16}$ through tailoring the inter and intramolecular forces.

\footnotetext{
${ }^{a}$ MTA-SZTE Lendület Biocolloids Research Group, Interdisciplinary Excellence Centre, Department of Physical Chemistry and Materials Science, University of Szeged, H-6720 Szeged, Hungary.E-mail: szistvan@chem.u-szeged.hu; Tel: +3662343255

${ }^{b}$ Institute of Chemical Sciences and Engineering, École Polytechnique Fédérale de Lausanne (EPFL), CH-1015 Lausanne, Switzerland

${ }^{c}$ Faculty of Chemistry and Chemical Technology, University of Ljubljana,

Večna pot 113, SI-1000 Ljubljana, Slovenia

$\dagger$ Electronic supplementary information (ESI) available. See DOI: 10.1039/ d0cp02805c

\$ These authors contributed equally to the work.
}

The origin of ion specific effects on surface forces has been systematically explored by optical tweezer, ${ }^{17}$ atomic force microscope ${ }^{18}$ and surface force apparatus ${ }^{19}$ in aqueous solutions of ILs and one of the main conclusions was that different affinity of the cations and anions for the surface may cause significant variation in the charge density upon ion adsorption. Consequently, this variation results in different extents of electrostatic repulsions between surfaces. Similar hypotheses were also reported on the basis of particle aggregation experiments in IL-water mixtures. ${ }^{9,20,21}$ Moreover, theoretical studies shed light on the fact that the interfacial assembly of IL ions mainly depends on the composition of the surface, concentration and structure of the ILs. ${ }^{10,22}$

The IL cations and anions may be arranged in series based on their effects on the surface charge and interaction forces, similar to the Hofmeister series of ions, which was originally constructed for protein destabilization by simple salts. ${ }^{21,23,24}$ Despite the fact that understanding the interfacial assembly of ILs is of huge importance during development of advanced materials in energy storage, ${ }^{25-27}$ electrochemistry ${ }^{28}$ and the preparation of novel catalysts, ${ }^{29}$ only a few studies dealt with the systematic assessment of the effect of IL constituents on the charge and aggregation features of particles. ${ }^{9,20,21}$ Another important aspect is that ion specific effects often hinder the application of IL mixtures to disperse and stabilize particles successfully. For instance, different affinity of the ions to the surfaces leads to asymmetric arrangement of the IL constituents near the surface, which may induce destabilization of the dispersions. Therefore, the possibility 
of masking interfacial ion specific effects would be beneficial in terms of gaining the stability of numerous systems. Nevertheless, to the best of our knowledge, no papers have been published on this topic yet.

In the present study, surface charges and colloidal stability of polymeric particles were investigated in the presence of aqueous IL solutions containing chloride $\left(\mathrm{Cl}^{-}\right)$, bromide $\left(\mathrm{Br}^{-}\right)$, nitrate $\left(\mathrm{NO}_{3}{ }^{-}\right)$ or acetate $\left(\mathrm{Ac}^{-}\right)$anions and the 1-butyl-3-methylimidazolium $\left(\mathrm{BMIM}^{+}\right)$cation. Two types of positively charged particles were studied, namely, polystyrene-based amidine latex (AL) and SL-IP-2 consisting of a sulfate latex (SL) functionalized with an imidazolium-based polymer (IP-2). In this way, ion specificity was explored with polymeric particles of the same sign of charge, but with different surface functionalities.

\section{Experimental}

\subsection{Materials}

Spherical polystyrene latex particles functionalized with sulfate (SL) or amidine (AL) groups were purchased from Thermo Fischer Scientific. The particle size and the charge density of the bare surfaces were determined by the manufacturer using transmission electron microscopy (TEM) and conductometric titration, respectively. The SL has a mean diameter of $430 \mathrm{~nm}$, a polydispersity expressed as coefficient of variation of $1.8 \%$, solid content of $8.1 \%(\mathrm{w} / \mathrm{v} \%)$, specific surface area of $1.3 \times 10^{5} \mathrm{~cm}^{2} \mathrm{~g}^{-1}$ and a charge density of $-12 \mathrm{mC} \mathrm{m}^{-2}$. In the case of the $\mathrm{AL}$, the corresponding quantities are $510 \mathrm{~nm}, 4.6 \%, 4.1 \%, 1.1 \times 10^{5} \mathrm{~cm}^{2} \mathrm{~g}^{-1}$ and $+197 \mathrm{mC} \mathrm{m}^{-2}$.

The polyimidazolium-based polymer (IP-2) was synthesized using a literature protocol. ${ }^{30}$ Briefly, 1,4-bis(chloromethyl)benzene and 1-(trimethylsilyl)imidazole were dissolved in acetonitrile in 1:1 molar ratio in a Schlenk-flask. The mixture was heated to reflux for $48 \mathrm{~h}$. The white solid product was removed by filtration, washed with acetonitrile and diethyl ether followed by drying under vacuum for $24 \mathrm{~h}$. All chemicals used for the synthesis are from Sigma-Aldrich. The successful synthesis was confirmed with FT-IR spectroscopy by identifying the characteristic vibration peaks of IP-2 at 1150, 1560 and $1625 \mathrm{~cm}^{-1}$ (for the spectrum see Fig. S1 in the $\mathrm{ESI} \dagger){ }^{30}$

Analytical grade potassium chloride ( $\mathrm{KCl})$ was purchased from VWR. The ILs, 1-butyl-3-methylimidazolium nitrate $\left(\mathrm{BMIMNO}_{3}\right.$, $\geq 95 \%$ ), 1-butyl-3-methylimidazolium chloride (BMIMCl, $\geq 98 \%$ ), 1-butyl-3-methylimidazolium acetate (BMIMAc, $\geq 95 \%$ ) and 1-butyl-3-methylimidazolium bromide (BMIMBr, >97\%) were purchased from Sigma-Aldrich. During sample preparation, ultrapure water was obtained from a Puranity TU+ (VWR) apparatus. The water and the salt solutions were filtered with $0.1 \mu \mathrm{m}$ syringe filters (Millex) to avoid dust contamination. All measurements were carried out at $25{ }^{\circ} \mathrm{C}$ and $\mathrm{pH}$ value of 4 set by $\mathrm{HCl}$ (VWR) stock solutions.

The IP-2-modified SL stock dispersion (denoted as SL-IP-2 thereafter) was prepared by simple mixing of proper volumes of IP-2 of $100 \mathrm{mg} \mathrm{L}^{-1}$ concentration, SL particle stock solution of $10 \mathrm{~g} \mathrm{~L}^{-1}$ and ultrapure water.

\subsection{Characterization of IP-2 and the ILs}

The FT-IR spectrum was recorded on a JASCO FT-IR-4700 spectrometer equipped with a DTGS detector in attenuated total reflectance mode. Spectral resolution was $4 \mathrm{~cm}^{-1}$ with 256 scans collected for the spectrum, which was recorded in the wavenumber range of $1800-1000 \mathrm{~cm}^{-1}$.

Refractive index and viscosity measurements were carried out with an Anton Paar Abbemat 3200 refractometer and a capillary viscometer, respectively. These data were used for the analysis of the light scattering measurements. For the data treatment, see Fig. S2a, b and Table S1 in the ESI. $\dagger$

\subsection{Electrophoresis}

Electrophoretic mobility was measured with a Litesizer 500 instrument (Anton Paar) equipped with a $40 \mathrm{~mW}$ semiconductor laser of $658 \mathrm{~nm}$ wavelength operating in the backscattering mode at a scattering angle of $175^{\circ}$. For the determination of the electrophoretic mobilities, $2.0 \mathrm{~mL}$ samples were prepared. In brief, $0.4 \mathrm{~mL}$ particle dispersions of $80 \mathrm{mg} \mathrm{L^{-1 }}$ or $0.2 \mathrm{~mL}$ of $100 \mathrm{mg} \mathrm{L}^{-1}$ concentration for $\mathrm{AL}$ and SL-IP-2, respectively, was added to $1.6 \mathrm{~mL}$ or $1.8 \mathrm{~mL}$ of solutions composed of ILs or $\mathrm{KCl}$ at appropriate concentrations. The samples were left to rest for $2 \mathrm{~h}$ at room temperature before measuring the electrophoretic mobilities, which occurred after 1 min equilibration time in the device. The reported values were the average of five individual measurements with a maximum standard deviation of $\pm 5 \mathrm{mV}$. The experiments were performed in $350 \mu \mathrm{L}$ volume $\Omega$-shaped plastic cuvettes or with the Univette (Anton Paar) accessory.

The measured electrophoretic mobility $(u)$ was converted to zeta potential $(\zeta)$ with the Smoluchowski equation as follows ${ }^{31}$

$$
\zeta=u \eta / \varepsilon_{0} \varepsilon
$$

where $\varepsilon_{0}$ is the dielectric permittivity of vacuum and $\varepsilon$ is the dielectric constant of water, with values of $8.854 \times 10^{-12} \mathrm{~F} \mathrm{~m}^{-1}$ and 78.5 , respectively. The charge density at the slip plane $(\sigma)$ was calculated according to the Debye-Hückel model ${ }^{32}$ as

$$
\sigma=\varepsilon \varepsilon_{0} \kappa \zeta
$$

where $\kappa$ is the inverse Debye length, which contains the contribution of the ionic species to the electrical double layer and can be calculated for the IL solutions as ${ }^{31}$

$$
\kappa=\sqrt{2 N_{\mathrm{A}} e^{2} c_{\mathrm{IL}} / \varepsilon_{0} \varepsilon k_{\mathrm{B}} T}
$$

where $N_{\mathrm{A}}$ is Avogadro's number, $e$ is the elementary charge, $k_{\mathrm{B}}$ is the Boltzmann constant and $T$ is the absolute temperature.

\subsection{Dynamic light scattering}

The hydrodynamic radius $\left(R_{\mathrm{h}}\right)$ of the particles was measured by dynamic light scattering (DLS) using a NIBS High-Performance Particle Sizer (ALV) equipped with a $3 \mathrm{~mW} \mathrm{He}-\mathrm{Ne}$ laser of $633 \mathrm{~nm}$ wavelength. The scattering angle was $173^{\circ}$, the correlation function was collected for $20 \mathrm{~s}$ and was fitted with the cumulant method $^{33}$ to obtain the decay rate constant $(\Gamma)$. The diffusion coefficient $(D)$ was calculated as 


$$
D=\Gamma / q^{2}
$$

where $q$ is the scattering vector, which can be estimated using the parameters of the experimental setup as follows

$$
q=(4 \pi n / \lambda) \sin (\Theta / 2)
$$

where $n$ is the refractive index of the medium, $\lambda$ is the wavelength of the laser beam and $\Theta$ is the scattering angle. The refractive indices of the IL solutions were determined and eqn (S1) (ESI $\dagger$ ) was used for data interpolation. The $R_{\mathrm{h}}$ was then calculated with Stokes-Einstein equation ${ }^{34}$ as

$$
R_{\mathrm{h}}=k_{\mathrm{B}} T / 6 \eta \pi D
$$

The viscosity data points were interpolated using eqn (S2) (ESI $\dagger$ ). Time-resolved DLS measurements were carried out to assess the possible aggregation processes in the samples. Typical results of these measurements, where 100-250 runs were performed depending on the speed of aggregation, are shown in Fig. S3 (ESI $\dagger$ ). During sample preparation, $2 \mathrm{~mL}$ dispersions were prepared. The ionic strength or IL dose was adjusted by mixing appropriate amounts of stock solutions and the time-resolved DLS measurements were initiated by adding the desired volume of particle stock dispersions to the solutions containing all other components. The particle concentration was $16 \mathrm{mg} \mathrm{L}^{-1}\left(2.18 \times 10^{14} \mathrm{~m}^{-3}\right)$ for $\mathrm{AL}$ and $10 \mathrm{mg} \mathrm{L}^{-1}\left(2.28 \times 10^{14} \mathrm{~m}^{-3}\right)$ for SL-IP-2. These particle doses were good compromises to avoid the formation of higher ranked aggregates and to maintain sufficient scattered intensity for the light scattering experiments. The aggregation rate coefficient (k) was calculated from the time dependent $R_{\mathrm{h}}$ values as ${ }^{33}$

$$
\left(1 / R_{\mathrm{h}}^{1}\right)\left(\mathrm{d} R_{\mathrm{h}} / \mathrm{d} t\right)=\left(I_{2} / 2 I_{1}\right)\left(1-\left(R_{\mathrm{h}}^{1} / R_{\mathrm{h}}^{2}\right)\right) k N_{0}
$$

where $t$ is the experiment time, $N_{0}$ is the number concentration of the particles, $R_{\mathrm{h}}^{1}$ and $R_{\mathrm{h}}^{2}$ are the hydrodynamic radius of the monomer and dimer, respectively. Note that only dimer formation is assumed until $40 \%$ increase in the hydrodynamic radius. ${ }^{33}$ The contribution of the form factors of the monomer $\left(I_{1}\right)$ and dimer $\left(I_{2}\right)$ to the scattered intensity was calculated with the theory developed by Rayleigh, Debye and Gans ${ }^{33}$

$$
I_{2} / 2 I_{1}=1+(\sin (2 R q) / 2 R q)
$$

where $R$ is the geometric radius of the particle. The colloidal stability of the samples was expressed in terms of stability ratio $(W)$, which can be calculated as

$$
W=k_{\text {fast }} / k
$$

where the $k_{\text {fast }}$ was determined in $1 \mathrm{M} \mathrm{KCl}$ solutions, in which the repulsive double layer forces vanish and the particles undergo rapid aggregation, as predicted by the theory of Derjaguin, Landau, Vervey and Overbeek (DLVO). ${ }^{32,35}$ The $k_{\text {fast }}$ values were found to be $5.70 \times 10^{-18} \mathrm{~m}^{3} \mathrm{~s}^{-1}$ and $3.33 \times 10^{-18} \mathrm{~m}^{3} \mathrm{~s}^{-1}$ for the $\mathrm{AL}$ and SL-IP-2 particles, respectively. These data are similar to the ones obtained for latex particles earlier in the presence of monovalent electrolytes. ${ }^{21,36}$ Note that the stability ratio is one in case of unstable dispersions, i.e., all particle collisions lead to dimer formation. Higher values, on the other hand, refer to slower aggregation and thus, to more stable samples. The standard error of stability ratio measurement is about $10 \%$.

The critical coagulation concentration (CCC) values were calculated from the $W$ versus $c_{\mathrm{IL}}$ plots with the following equation $^{37}$

$$
1 / W=1 /\left(1+C C C / c_{\mathrm{IL}}\right)^{\beta}
$$

where $\beta$ can be obtained from the slow aggregation regime (i.e., before the CCC) of the $W$ versus $c_{\mathrm{IL}}$ graphs as

$$
\beta=\mathrm{d} \log (1 / W) / \mathrm{d} \log c_{\mathrm{IL}}
$$

To compare experimental and theoretical CCC data, DLVO theory, which assumes that the total interaction energy can be considered as a superposition of the repulsive electrostatic double layer potential energy and the attractive van der Waals potential energy, was used to estimate the CCC as follows ${ }^{36}$

$$
\mathrm{CCC}=\left(0.365 / N_{\mathrm{A}} L_{\mathrm{B}}\right)\left(H \varepsilon_{0} \varepsilon\right)^{-2 / 3} \sigma^{4 / 3}
$$

where $H$ is the Hamaker constant $\left(1.00 \times 10^{-21} \mathrm{~J}\right.$ was used for both AL and SL-IP-2 particles) and $L_{\mathrm{B}}$ is the Bjerrum length, which was calculated as

$$
L_{\mathrm{B}}=e^{2} / 4 \pi \varepsilon_{0} \varepsilon k_{\mathrm{B}} T
$$

Using the model above, it was assumed that the energy barrier vanishes at the CCC and attractive interparticle forces predominate beyond this concentration.

\section{Results and discussion}

\subsection{Functionalization of SL with IP-2}

The AL particles are commercially available, whereas SL-IP-2 particles were prepared by adsorbing a saturated IP-2 layer on the oppositely charged SL. To determine the polymer dose necessary to fully coat the SL surface, the zeta potential values were determined at different IP-2 doses (Fig. 1).

The SL particles are negatively charged at low IP-2 doses due to the presence of ionized sulfate functional groups. Increasing the amount of IP-2 led to charge neutralization and subsequent overcharging of the SL particles. The zeta potential values remain constant at high IP-2 doses, at which the surface of the particles contains a saturated IP-2 layer. These results resemble to other systems, where polyelectrolytes were adsorbed on oppositely charged latex particles. ${ }^{38-40}$

The aggregation tendencies were assessed by determining stability ratio values under the same experimental conditions as used for the potential measurements. Note that the stability ratio of unity refers to diffusion controlled rapid particle aggregation, while higher values indicate a decrease in the aggregation rates. The data shown in Fig. 1 suggest that rapid particle aggregation occurred near the charge neutralization point, while stable dispersions were observed far from it. Most importantly, particle aggregation is negligible at high doses.

Very similar observations were taken in systems containing oppositely charged latexes and polyelectrolytes, ${ }^{38-40}$ but this is the first report, which involves a polyimidazolium compound. 


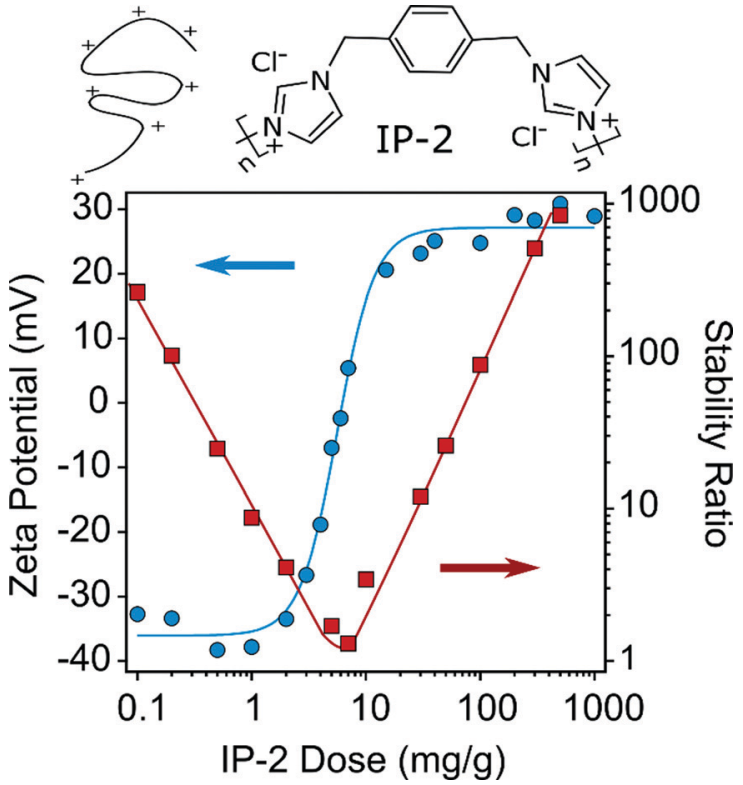

Fig. 1 Zeta potential (circles, left axis) and stability ratio (squares, right axis) values of SL particles as a function of the IP-2 dose at $\mathrm{pH} 4$ and $10 \mathrm{mM}$ ionic strength adjusted by $\mathrm{KCl}$. Note that the inverse of the stability ratio is equal to the fraction of particle collisions, which results in dimer formation. The $\mathrm{mg} / \mathrm{g}$ unit refers to $\mathrm{mg}$ IP-2 per one gram of SL. The lines serve to guide the eye. The arrows indicate the axis, to which potential (blue arrow) and stability ratio (red arrow) data points belong. The upper part shows the structure of IP-2.

Based on the above results, a dose of $500 \mathrm{mg} \mathrm{g}^{-1}$ was chosen (SL-IP-2) for the further studies, since under this condition the particle surface was saturated with IP-2 and stable dispersion was formed.

\subsection{Charge and aggregation features of AL and SL-IP-2 particles in the presence of ILs}

To investigate the specific interfacial effects of IL constituents, the zeta potentials of $\mathrm{AL}$ particles were measured first in aqueous solutions of BMIMAc, $\mathrm{BMIMNO}_{3}$, BMIMBr and BMIMCl in a wide range of IL concentrations. The results are shown in Fig. 2a. Note that the anions are the counterions and the $\mathrm{BMIM}^{+}$ cation acts as co-ion in respect to the positively charged particles. In general, the zeta potential values decreased with the IL concentrations, similar to the case of indifferent $\mathrm{KCl}$ electrolyte (Fig. S4a, ESI $\dagger$ ).

The surface charge densities were calculated from the salt concentration-dependent potential data using the Debye-Hückel model (eqn (2)). ${ }^{41}$ The values (Table S2, ESI $\dagger$ ) decreased in the order $\mathrm{Cl}^{-}>\mathrm{Br}^{-}>\mathrm{NO}_{3}{ }^{-}>\mathrm{Ac}^{-}$indicating specific adsorption of the ions. Note that these charge densities were measured at the slip plane, therefore their magnitudes are much lower than the charge density of the bare surface determined in conductometric measurements by the manufacturer. Such a sequence agrees well with the reversed Hofmeister series, which classifies anions by their affinity towards positively charged hydrophobic surfaces. ${ }^{42}$ Accordingly, the adsorption of the well-hydrated $\mathrm{Cl}^{-}$is negligible, while $\mathrm{Ac}^{-}$adsorbs strongly on the oppositely charged surface.
For the SL-IP-2 system, nevertheless, the zeta potentials were the same within the experimental error (Fig. 2b). After an intermediate maximum due to the electrokinetic effect, ${ }^{43}$ the potentials decreased with increasing the IL concentrations in all cases, but no specific adsorption of the IL anions was detected. This result is in a striking contrast to the findings for the AL systems. In addition, specific anion adsorption on positively charged bare latexes ${ }^{21,36}$ and the ones functionalized with positively charged polyelectrolytes ${ }^{39}$ was reported in the past. Therefore, the insensitivity of the surface charge to the chemical composition of the surrounding anions in the SL-IP-2 systems is rather surprising. The different affinity of the anions to cationic surfaces was explained by their different level of hydration, i.e., less hydrated anions tend to adsorb on hydrophobic surfaces, while this effect was not observed with the SL-IP-2 particles, most likely due to the less hydrophobic nature of their surface.

The stability ratios were determined by DLS to assess the particle aggregation processes in the IL solutions. The experimental conditions (e.g., pH value, particle and IL concentration range) were the same as in the zeta potential study in order to allow the direct comparison of the results. For the AL systems, the stability ratios follow the same generic trend as a function of the IL concentration, i.e., their values were high (slow aggregation) at low salt levels and decreased with increasing the IL loading and became unity (fast aggregation) at high IL concentrations (Fig. 2c). The threshold value, which separate the slow and fast aggregation regimes, is the so-called CCC, which was calculated by eqn (10).

These results are similar to the ones reported earlier for latex particle dispersions in simple inorganic salt solutions (see the case of $\mathrm{KCl}$ in Fig. S4b, ESI $\dagger$ ) and they are in line with the prediction of the DLVO theory. ${ }^{35,44}$ Accordingly, the overall forces acting between charged particles are the sum of the repulsive electrical double layer and the attractive van der Waals forces. Increasing the salt concentration leads to the weakening of the electrostatic repulsion by salt screening and thus, the particles rapidly aggregate after the CCC due to the predominating attractive forces. This explanation is also evident from the very low zeta potential values measured at the CCC (Table S2, ESI $\dagger$ ).

Although the generic trend in the stability ratios was very similar in all systems containing AL particles, the CCC values were different (Fig. 3a). This result cannot be explained by the DLVO theory since it predicts the same CCC, once a particle is dispersed in different types of monovalent salt solutions. The CCC values follow the order $\mathrm{Cl}^{-}>\mathrm{Br}^{-}>\mathrm{NO}_{3}{ }^{-}>\mathrm{Ac}^{-}$, in accordance with the surface charge density data determined in the same systems. Again, this order is in line with the suggestion of the reversed Hofmeister series for positively charged hydrophobic particles. ${ }^{36,42}$

For the SL-IP-2 particles, nevertheless, the measured stability ratio values were identical within the experimental error for BMIMAc, BMIMNO ${ }_{3}$, BMIMBr and BMIMCl (Fig. 2d) and thus, the onset of rapid particle aggregation was located at the same CCC for all systems. This finding is in striking contrast to earlier results with latex particles functionalized with a positively charged natural polyelectrolyte, where the presence of different inorganic salts led to different CCC values. ${ }^{39}$ More importantly, we are not 



Fig. 2 Zeta potentials (AL (a) and SL-IP-2 (b)) as well as stability ratios (AL (c) and SL-IP-2 (d)) as a function of IL concentration at pH 4. The functions represented by lines in (a) and (b) were calculated using eqn (2) and in (c) with eqn (10).
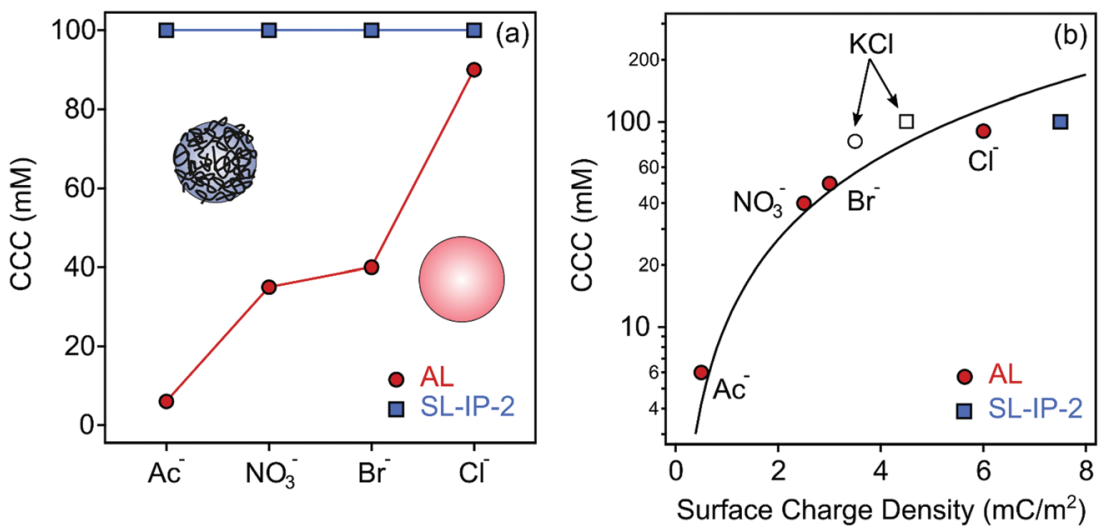

Fig. 3 CCC values of AL (red circles) and SL-IP-2 (blue square) determined in the presence of ILs (a) and CCC versus the charge density data (b). The empty symbols indicate the data measured with $\mathrm{KCl}$. The lines in (a) were added merely to guide the eyes, while in (b), it was calculated using eqn (12).

aware of findings similar to the present one, which demonstrate that polyelectrolyte coating can be used to abolish ion specific effects on particle aggregation.

Besides, the tendency in the stability ratios was remarkably different from the AL samples and intermediate plateaus were discovered around $10 \mathrm{mM}$ IL concentrations. The term "plateau" refers to the fact that the decrease of the stability ratios was not linear, but curved in the slow aggregation regime. This is rather unusual for latex particles and most likely originates from the swelling of the adsorbed IP-2 layer, which is indeed predicted in the same ionic strength range as the location of the plateaus. ${ }^{45}$ Such a swelling gives rise to the formation of polyelectrolyte tails and loops on the surface and subsequently, to the rise of stabilizing steric forces ${ }^{12}$ to a minor extent (the main interparticle forces are still of DLVO origin). The increment in the stability ratios in this concentration range is due to the presence of these additional stabilization forces. An important note is that the height and the location of these plateaus are the same for all $\mathrm{IL}$ solutions. Notably, no ion specific effects could be detected for the aggregation of SL-IP-2 particles. 


\subsection{Origin of interparticle forces}

Given the very similar surface charge density and CCC values for the AL and SL-IP-2 particles in inert $\mathrm{KCl}$ electrolyte, the striking contrast in the charge and aggregation features in IL solutions is very surprising. Note that deviations in the zeta potentials (Fig. S5a-d, ESI $\dagger$ ) and stability ratios (Fig. S6a-d, ESI $\dagger$ ) measured in the same IL solution for AL and SL-IP-2 particles are larger in the sequence $\mathrm{Cl}^{-}>\mathrm{Br}^{-}>\mathrm{NO}_{3}^{-}>\mathrm{Ac}^{-}$. These results show that ion specific effects can be abolished by surface functionalization of polyimidazolium.

To further clarify the origin of the interparticle forces for both AL and SL-IP-2 particles dispersed in IL solutions, the experimental CCC values were plotted against the surface charge density data and compared to the CCC calculated using the DLVO theory (eqn (12)). ${ }^{36}$ The data shown in Fig. 3b indicate that the calculated and experimental CCC values are in good agreement, which indicates the presence of DLVO-type forces. However, the ion specific adsorption led to different surface charge densities for AL, which decrease in the order $\mathrm{Cl}^{-}>\mathrm{Br}^{-}>$ $\mathrm{NO}_{3}{ }^{-}>\mathrm{Ac}^{-}$leading to weaker electrical double layer repulsion. Since the van der Waals forces are always present and their strength does not depend on the ionic strength, the CCC values decrease in the above order due to the weakening of the repulsive double layer forces. In other words, DLVO forces govern the aggregation mechanism, while the CCC is determined by specific ion adsorption.

However, such a specific ion effect is absent for SL-IP-2 particles. The basic phenomenon behind this result most probably originates from the hydration level of the surface and the counterions. It was reported in other latex systems that weakly hydrated ions are of higher affinity to hydrophobic surfaces, i.e., they adsorb stronger on hydrophobic latexes, ${ }^{21}$ similar to the AL particles in the present study. Since the same counterions were applied with the SL-IP-2 particles, the lack of ion specific effects implies that the hydrophobic character of SL weakened upon surface functionalization by IP-2 and thus, the hydration level-based affinity of the counterions is not pronounced. The reason for the decreased hydrophobicity can be that stacking interaction took place between the imidazolium groups of $\mathrm{BMIM}^{+}$and IP-2 leading to an accumulation of the $\mathrm{BMIM}^{+}$molecules on the surface and to the formation of a more ionic interfacial environment. Such a stacking interaction was reported earlier based on electronic structure analysis. ${ }^{46}$

Although the present study focuses on polymeric particles, similar coating can be also possible on other surfaces used in ILs. ${ }^{6,11,13,26,27}$ This would be a promising strategy to develop ionophobic surfaces, however, the experimental conditions (e.g., $\mathrm{pH}$, polymer concentration and ionic strength) must be precisely optimized before the coating procedure.

\section{Conclusions}

To conclude, it was successfully demonstrated that anion specific effects on the surface charge and aggregation of $\mathrm{AL}$ particles are important and that the tendency can be predicted by the reversed Hofmeister series of anions for positively charged hydrophobic surfaces. The ion specific adsorption led to a decrease in the surface charge density and thus, lower CCC values were measured for strongly adsorbing anions such as $\mathrm{Ac}^{-}$. The ion specificity plays an important role in the adsorption process, while the aggregation mechanism and the major interparticle forces could be described with the DLVO theory. These ion specific interactions, however, can be masked by functionalization of the surface with polyimidazolium, after which the anions behave like indifferent salt constituents giving rise to the same colloidal stability of the SL-IP-2 particles in different ILs. These results are especially important for the design of particle-IL dispersion of desired surface charge and aggregation characteristics.

\section{Conflicts of interest}

There are no conflicts to declare.

\section{Acknowledgements}

The authors acknowledge financial support from the Hungarian National Research, Development and Innovation Office (SNN131558), the Slovenian Research Agency (research core funding No. P1-0201 and the project No. N1-0139 'Delamination of Layered Materials and Structure-Dynamics Relationship in Green Solvents') and the Ministry of Human Capacities of Hungary (20391-3/2018/FEKUSTRAT). The support from the University of Szeged Open Access Fund (5019) is gratefully acknowledged.

\section{References}

1 R. Hayes, G. G. Warr and R. Atkin, Chem. Rev., 2015, 115, 6357-6426.

2 Z. P. Zheng, W. H. Fan, S. Roy, K. Mazur, A. Nazet, R. Buchner, M. Bonn and J. Hunger, Angew. Chem., Int. Ed., 2015, 54, 687-690.

3 R. D. Rogers and K. R. Seddon, Science, 2003, 302, 792-793.

4 M. Taskin, A. Cognigni, R. Zirbs, E. Reimhult and K. Bica, RSC Adv., 2017, 7, 41144-41151.

5 K. Z. Donato, L. Matejka, R. S. Mauler and R. K. Donato, Colloids Interfaces, 2017, 1, 5.

6 D. A. Beattie, R. M. Espinosa-Marzal, T. T. M. Ho, M. N. Popescu, J. Ralston, C. J. E. Richard, P. M. F. Sellapperumage and M. Krasowska, J. Phys. Chem. C, 2013, 117, 23676-23684.

7 R. M. Espinosa-Marzal, A. Arcifa, A. Rossi and N. D. Spencer, J. Phys. Chem. Lett., 2014, 5, 179-184.

8 A. Elbourne, B. McLean, K. Voitchovsky, G. G. Warr and R. Atkin, J. Phys. Chem. Lett., 2016, 7, 3118-3122.

9 I. Szilagyi, T. Szabo, A. Desert, G. Trefalt, T. Oncsik and M. Borkovec, Phys. Chem. Chem. Phys., 2014, 16, 9515-9524.

10 A. Podgorsek, A. S. Pensado, C. C. Santini, M. F. C. Gomes and A. A. H. Padua, J. Phys. Chem. C, 2013, 117, 3537-3547.

11 E. Vanecht, K. Binnemans, S. Patskovsky, M. Meunier, J. W. Seo, L. Stappers and J. Fransaer, Phys. Chem. Chem. Phys., 2012, 14, 5662-5671. 
12 K. Ueno, A. Inaba, M. Kondoh and M. Watanabe, Langmuir, 2008, 24, 5253-5259.

13 P. P. Arquilliere, I. S. Helgadottir, C. C. Santini, P. H. Haumesser, M. Aouine, L. Massin and J. L. Rousset, Top. Catal., 2013, 56, 1192-1198.

14 D. Constantinescu, H. Weingartner and C. Herrmann, Angew. Chem., Int. Ed., 2007, 46, 8887-8889.

15 N. Debeljuh, C. J. Barrow and N. Byrne, Phys. Chem. Chem. Phys., 2011, 13, 16534-16536.

16 W. L. Li and P. Y. Wu, Polym. Chem., 2014, 5, 761-770.

17 M. M. Elmahdy, C. Gutsche and F. Kremer, J. Phys. Chem. C, 2010, 114, 19452-19458.

18 V. Valmacco, G. Trefalt, P. Maroni and M. Borkovec, Phys. Chem. Chem. Phys., 2015, 17, 16553-16559.

19 M. W. Han and R. M. Espinosa-Marzal, ACS Appl. Mater. Interfaces, 2019, 11, 33465-33477.

20 P. Rouster, M. Pavlovic, T. Cao, B. Katana and I. Szilagyi, J. Phys. Chem. C, 2019, 123, 12966-12974.

21 T. Oncsik, A. Desert, G. Trefalt, M. Borkovec and I. Szilagyi, Phys. Chem. Chem. Phys., 2016, 18, 7511-7520.

22 T. Kobayashi, A. Kemna, M. Fyta, B. Braunschweig and J. Smiatek, J. Phys. Chem. C, 2019, 123, 13795-13803.

23 W. Kunz, J. Henle and B. W. Ninham, Curr. Opin. Colloid Interface Sci., 2004, 9, 19-37.

24 A. A. Tietze, F. Bordusa, R. Giernoth, D. Imhof, T. Lenzer, A. Maass, C. Mrestani-Klaus, I. Neundorf, K. Oum, D. Reith and A. Stark, ChemPhysChem, 2013, 14, 4044-4064.

25 D. R. MacFarlane, M. Forsyth, P. C. Howlett, M. Kar, S. Passerini, J. M. Pringle, H. Ohno, M. Watanabe, F. Yan, W. J. Zheng, S. G. Zhang and J. Zhang, Nat. Rev. Mater., 2016, 1, 15005.

26 S. Kondrat and A. A. Kornyshev, Nanoscale Horiz., 2016, 1, 45-52.

27 S. Kondrat, P. Wu, R. Qiao and A. A. Kornyshev, Nat. Mater., 2014, 13, 387-393.

28 X. W. Mao, P. Brown, C. Cervinka, G. Hazell, H. Li, Y. Y. Ren, D. Chen, R. Atkin, J. Eastoe, I. Grillo, A. A. H. Padua, M. F. C. Gomes and T. A. Hatton, Nat. Mater., 2019, 18, 1350-1357.
29 X. Xu, Y. Li, Y. T. Gong, P. F. Zhang, H. R. Li and Y. Wang, J. Am. Chem. Soc., 2012, 134, 16987-16990.

30 W. Zhong, F. D. Bobbink, Z. F. Fei and P. J. Dyson, ChemSusChem, 2017, 10, 2728-2735.

31 A. V. Delgado, F. Gonzalez-Caballero, R. J. Hunter, L. K. Koopal and J. Lyklema, J. Colloid Interface Sci., 2007, 309, 194-224.

32 G. Trefalt, I. Szilagyi and M. Borkovec, J. Colloid Interface Sci., 2013, 406, 111-120.

33 H. Holthoff, S. U. Egelhaaf, M. Borkovec, P. Schurtenberger and H. Sticher, Langmuir, 1996, 12, 5541-5549.

34 P. A. Hassan, S. Rana and G. Verma, Langmuir, 2015, 31, 3-12.

35 B. Derjaguin and L. D. Landau, Acta Phys. Chim., 1941, 14, 633-662.

36 T. Oncsik, G. Trefalt, M. Borkovec and I. Szilagyi, Langmuir, 2015, 31, 3799-3807.

37 D. Grolimund, M. Elimelech and M. Borkovec, Colloids Surf., A, 2001, 191, 179-188.

38 I. Szilagyi, G. Trefalt, A. Tiraferri, P. Maroni and M. Borkovec, Soft Matter, 2014, 10, 2479-2502.

39 T. Lopez-Leon, A. B. Jodar-Reyes, J. L. Ortega-Vinuesa and D. Bastos-Gonzalez, J. Colloid Interface Sci., 2005, 284, 139-148.

40 J. Hierrezuelo, A. Vaccaro and M. Borkovec, J. Colloid Interface Sci., 2010, 347, 202-208.

41 W. B. Russel, D. A. Saville and W. R. Schowalter, Colloidal dispersions, Cambridge University Press, Cambridge, 1989.

42 N. Schwierz, D. Horinek and R. R. Netz, Langmuir, 2010, 26, 7370-7379.

43 M. Borkovec, S. H. Behrens and M. Semmler, Langmuir, 2000, 16, 5209-5212.

44 E. J. W. Verwey and J. T. G. Overbeek, Theory of stability of lyophobic colloids, Elsevier, Amsterdam, 1948.

45 J. Hierrezuelo, I. Szilagyi, A. Vaccaro and M. Borkovec, Macromolecules, 2010, 43, 9108-9116.

46 R. P. Matthews, T. Welton and P. A. Hunt, Phys. Chem. Chem. Phys., 2015, 17, 14437-14453. 